\title{
PREFACE
}

\section{Urology and Gynecology}

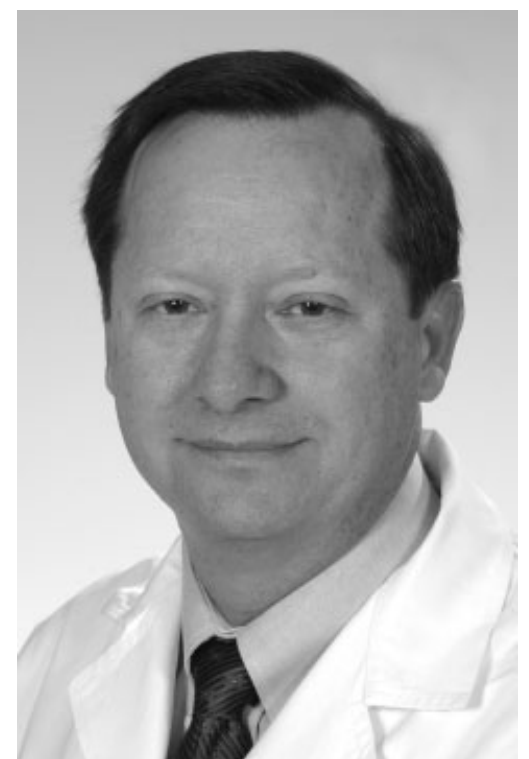

Colorectal surgeons work frequently in the pelvis, which contains urologic and gynecologic organs. Abnormalities of these organs are commonly encountered and unintentional and inadvertent injury can also occur. A multidisciplinary approach is exceptionally helpful in obtaining optimal results. However, valuable consultants are not always available and the colorectal surgeon needs a familiarity with pelvic organ management. This issue of Clinics provides insight on several important urologic and gynecologic topics.

This issue starts with a primer on adnexal masses and malignancies written by the Ochsner gynecologic oncologic surgeons Drs. Kline and Bazzett-Matabele. Next, Drs. Slaughter and Gala, from the Ochsner Gynecology Department, discuss the common problem of endometriosis.

Pregnant patients suffer from many of the gastrointestinal problems experienced by other patients. How- ever, their pregnant status can complicate evaluation and management. Drs. Longo, Moore, Canzoneri, and Robichaux from the Ochsner Obstetrics Department discuss the presentation, diagnosis, and treatment of several common gastrointestinal conditions experienced by pregnant patients.

Rectocele is a common condition treated by colorectal and gynecologic surgeons. Dr. Allen and I present a review. Drs. deBeche-Adams and Bohl from the Ochsner Colon and Rectal Surgery Department discuss their approach to surgical management of rectovaginal fistulas.

Finally, Drs. Delacroix and Winters from the Louisiana State University Department of Urology present three articles. They start with a discussion of the recognition and management of urinary tract injuries. Management of several malignant and benign conditions requires removal of the bladder. Reconstruction and replacement options can be complex and it is important that colorectal surgeons are familiar with the procedures available for this challenging problem. Their second article discusses these procedures. Any pelvic surgery has the potential for postoperative voiding dysfunction. The third urologic article presents a review of the evaluation and treatment of this problem.

Editing this issue has been a gratifying experience as it covers important aspects of our complex colorectal practices. Working in a large multidisciplinary group practice, I and my patients benefit from the expertise of knowledgeable and respected colleagues on a daily basis. The editors and I thank them for their additional assistance in producing this issue of Clinics. Their willingness to take time from their demanding practices and even more important limited family time to contribute to this issue is sincerely appreciated

David E. Beck, M.D. ${ }^{1}$

Guest Editor

\footnotetext{
${ }^{1}$ Department of Colon and Rectal Surgery, Ochsner Clinic Foundation, New Orleans, Louisiana.

Address for correspondence and reprint requests: David E. Beck, M.D., Department of Colon and Rectal Surgery, Ochsner Clinic Foundation, 1514 Jefferson Hwy., New Orleans, LA 70121 (e-mail: dbeckmd@aol.com).
}

Urology and Gynecology; Guest Editor, David E. Beck, M.D.

Clin Colon Rectal Surg 2010;23:61-62. Copyright (C) 2010 by Thieme Medical Publishers, Inc., 333 Seventh Avenue, New York, NY 10001, USA. Tel: +1(212) 584-4662.

DOI: http://dx.doi.org/10.1055/s-0030-1254291.

ISSN 1531-0043. 